\title{
La Pobreza en El Salvador un análisis sociológico. 2003
}

Por: Norma Mot.jna

\author{
Dedicada a los rostros invisibilizados \\ Para las personas ser pobre significa: \\ "La forma en que vivimos", "No tenernada, ni para trabajary vivir de lo que Diosy la gente me da", \\ "Vivir en zonas de al to riesgo y no tener dinero para comer", "Gastar más de lo que se gana", \\ "Manzener a los hijos desnudos y con hambre", "No poder pagar consultas médicas, es esforzarse duro \\ para poder obtener un ingreso"
}

\section{Introducción}

L a pobreza sigue siendo uno de los retos más grandes de la humanidad del nuevo siglo $y$, por ende, caracterizarla es de suma importancia. En la actualidad existe una diversidad de métodos para medirla: el del ingreso, el de las necesidad básicas, el de capacidad y también vista como exclusión social, entendida ésta, como la incapacidad para participar en la vida económica, culrural y política, aspectos que contribuyen a la reproducción material y espiritual de la vida humana. A simismo, se pueden distinguir diferentes niveles de pobreza, a saber: pobreza histórica, existencia histórica de sectores excluidos; pobreza de oportunidades, existencia efecriva de un sector excluido del ámbito económico; pobreza culcural, carecer o no tener acceso a fuentes que alimenten la cultura, la literatura científica, literaria, música, teatro, entre otros aspectos; pobreza recnológica, la carencia de tecnología apropiada para el desarrollo integral de las tareas normales de una comunidad.

En la definición de pobreza existe coincidencia, de analistas e instiruciones, en decir que los pobres son aquellos que tienen algún tipo de privación para su reproducción y tienden a establecerla de diferente forma. Para el caso, el PNUD ve la pobreza a través de su índice de desarrollo humano en el que incluye tres componentes básicos: la longevidad (mayor vida), los conocimientos (un minimo de educación) y el acceso a niveles de vida decentes. Para el caso, El Salvador se encuentra en un desarrollo humano medio, ocupando el lugar número 105.

El Banco Mundial concibe a los pobres como aquellas personas que crónicamente padecen de hambre y los clasifica en tres categorias: "los pobres de Dios" (los incapacitados, viudos, sin hijos), "los pobres sin recursos", y entre los dos extremos "los que sufren privaciones porque viven al dia, pero son trabajadores". En la lucha por erradicarla, en la
Cumbre del Milenio se establecieron las siguientes metas': 1) Erradicar el hambre y la pobreza extrema: el porcentaje de la población que vive con ingresos inferiores a 1 dólar por día es de $21.4 \%$ entre 1990 y 2001; 2) Alcanzar la educación primaria universal: estudiantes que comienzan primer grado y llegan al quinto grado durante 1990 y 1991 fue del 75\%; 3) Promover la igualdad de género y el empoderamiento de las mujeres: la relación entre niñas y niños en educación primaria para $2000-01$ fue de $0.93 \%$, proporción de mujeres entre los empleados remunerados en el sector no agrícola fue de $31 \%$ para el mismo período; 4) Reducir la mortalidad infantil: las tasas de mortalidad inf antil (por mil nacimienros vivos) para 1990 y 2001 fueron de 46 y 33 respectivamente; 5) Mejorar la salud materna; 6) Combatir el VIH/SIDA, la malaria y otras enfermedades, 7) Asegurar la sostenibilidad ambiental: la proporción de la superficie de las tierras cubiertas por bosques para los años 1990 y 2000 fue de 9.3 y 5.8 respecrivamente; $y$. 8) Desarrollar una sociedad global: el servicio de la deuda total se ha reducido, entre 1990 a 2001 , ha pasado de representar el $18,2 \%$ de las exportaciones a $7,4 \%$.

De tal manera, un individuo en su lucha por sobrevivir se enf renta $y$ condiciona por diferentes aspectos que se vinculan con su entorno cultural comunitario e institucional, y su

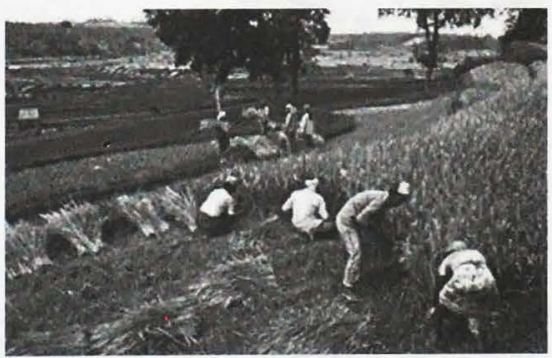

Agosto 2004, $\mathrm{N}^{\circ} .32$ 
interacción social lleva consigo un proceso de control social que, a su vez, posibilita el cambio o el estancamiento a través del riempo. En esta sobrevivencia, no se puede dejar de lado la cultura de la pobreza, en la cual los pobres adoptan una estrategia para sobrevivir a base de una economía casera, en una situación de escasas oportunidades especialmente para el trabajador no calificado y desempleado, que lo llevan a sueldos muy bajos o nulos. La existencia y predominio de una escala de valores de la clase dominante (que posee riquezas y propiedades) sobre la clase carente (con ninguna - casi nula posibilidad de ascender porque se encuentra sumergida en una vida cortoplacista) lleva a un predominio de acritudes de adapración e individualismoante un ambiente y sistema que lo sumerge en un ciclo de carencias y oportunidades.

Asi, la Dirección de Investigación Socioeconómica realizó dos investigaciones acerca de la pobreza. En el año 2002 se realizó una abstracción teórica del tema en el que se incluian aspectos económicos, sociales, culcurales y antropológicos y

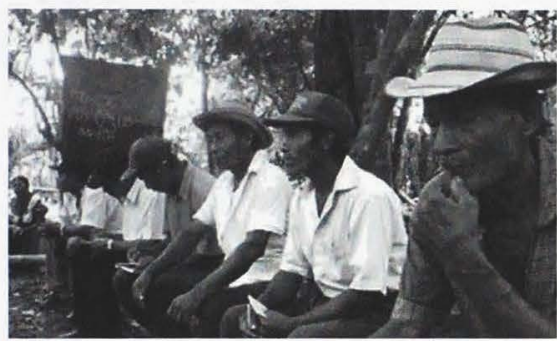

el estudio del caso de la comunidad "Las Palmas", que se encuentra ubicada al norponiente de San Salvador, detrás de las instalaciones de la Feria Internacional. Para el año 2003, se amplia la investigación de campo, se realiza la encuesta a diferentes municipios del pais. El fin de ambos estudios es tipificar a los pobres y recoger la percepción que ellos mismos tienen de sí y como tratan o como quisieran solventar sus condiciones; se realiza un análisis más allá de los tradicionalmente realizados por las diferentes insrituciones, al incluir codoslos aspectosque afecran la vida diaria de las familias: condiciones de vida, siruación económica, educación, y percepción antropológica y cultural.

\section{Diseño del instrumento}

Se realizó una encuesta en 13 municipios de 6 departamentos de El Salvador: 1) San Salvador: Ciudad Delgado, San Salvador, San Martín, Soyapango, Ilopango, Cuscatancingo; 2) Chalatenango: Tejutla, Chalatenango; 3) San Vicente: Apastepeque, San Vicente; 4) Sonsonate: Izalco;

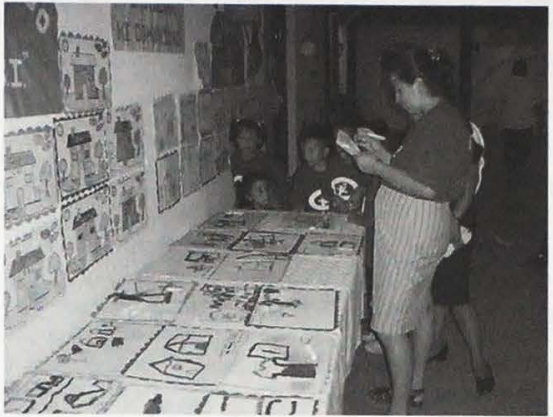

5) La Unión: La Unión; 6) Morazán: Morazán. Aplicándose 1544 instrumentos validos con 277 item, cuya muestra se eligió aleatoriamente para poblaciones infiniras. El nivel de error fue del $10 \%$. La información fue procesada en el programa estadístico para las ciencias sociales SPSS versión 10 para Windows, se crearon 4 bases de datos que contienen la información acerca de: datos de los muertos que los diferentes hogares habian suf rido, los proyectos que se daban en las diferentes comunidades visitadas, información demográfica (edades, nivel educativo, estado civil, escolaridad, situación de empleo) de cada grupo familiar en forma individual y finalmente la s variables socioeconómicas $y$ antropológicas en el que se recoge la información sobre la economia familiar y la visión de las personas acerca de la pobreza. El instrumento se dividió en 4 Secciones: a) Datos generales; b) Aspectos sociales; c) Aspectos Económicos; d) Aspectos antropológicos. Recolectando información a cerca de caracrerísticas socio-demográficas, educarivas, condiciones de vivienda, condiciones económicas, salud y nutrición, y finalmente características antropológicas. Es de saber que los lugares a donde se aplicó el instrumento se concibieron a priori como zonas pobres. Dos de los sirios tenían la peculiaridad de contar con población indigena: Huiscoyolate en Izalco, y Las Delicias en San Martín.

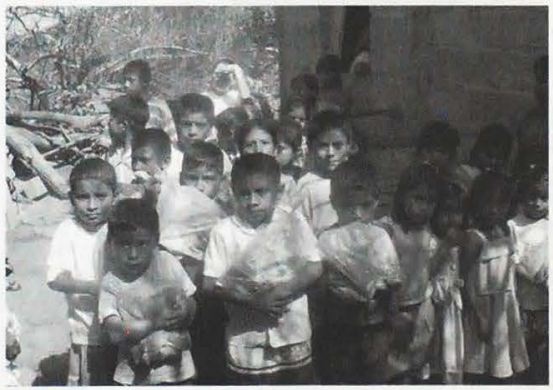

Agosto 2004, $\mathrm{N}^{\circ} .32$ 
Caracteristicas demográficas, educativa, salud, ingreso y empleo

Z xistió una correspondencia entre la población encuestada Ey los datos generales de la Encuesta de Hogares y Propósitos Múltiples (EHPM), en cuanto a la información general demográfica se refiere. Se entrevistaron 1544 hogares en los cuales el promedio familiar fue de 4 personas. Las mujeres representaron el $53,1 \%$ y los hombres el $46,1 \%$, de éstos el $55,1 \%$ correspondió a la zona rural y el $44,9 \%$ a la urbana. La unidad básica familiar era la familia compuesta por madre, padre e hijos, y en segunda instancia familias ampliadas. La mayor parte de la población era joven (entre niños, adolescentes y adultos jóvenes, entre 0 y 30 años) En relación a la situación civil, el $26,8 \%$ eran solteros, el 36,2\% acompañados y casados. El nivel educativo que prevaleció fue bajo y en forma piramidal, siendo la primaria el de mayor proporción y el técnico el menor. Se encontraron dos caracteristicas principales para no asistir a la escuela y fueron las siguientes: la situación económica y los problemas familiares.

Existe una relación entre educación, empleo e ingreso, de ahí que los resultados de la encuesta no fueran la excepción. Más de la mitad de la población no trabajaba por tres razones: eran exclusivamente estudiantes, infantes y amas de casa; es decir, población económicamente inacriva. La mayor parte de la población se encontraba en el sector servicio, incluyendo dueños de negocios; $y$, en menor proporción, en el sector productivo. Los ingresos percibidos también tuvieron una forma piramidal, menos del $10 \%$ percibía más de US $\$ 342.85$; y, más del $60 \%$ menos de US \$228.57 que es mas bajo al costo de la canasta familiar establecida en la EHPM del año 2002 (US \$254.07) La situación se vuelve más delicada si se considera la media del ingreso de los hogares (US \$184.93), reniendo el 37,7\% de los hogares en extrema pobreza y $35,6 \%$ en pobreza relativa, resultados mayores a

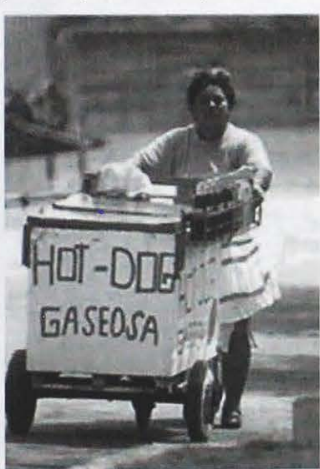

los establecidos por la EHPM.

Otro método de medir la pobreza es a través del ingreso per cápita, pese a que este puede ser el dato menos confiable debido a la nivelación del ingreso total por los hogares cuya renta es alca, ejemplo: el ingreso por hogar mensual fue de US \$161.91 y el per cápita de US \$35.68.

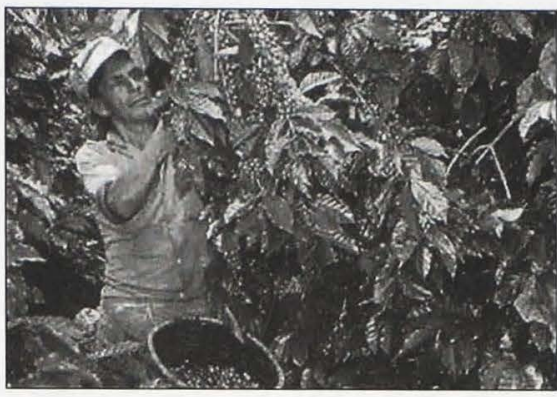

A nalistas señalan que la economia del pais se sostiene por las remesas familiares; no obstante, la mayoría de la población entrevistada no percibía ingresos en ese concepto.

\section{Características de la cultura de la pobreza}

Aspectos psico-sociales, referidos a los sentimientos de marginalidad, a la incorporación temprana al mercado laboral y al abandono de la escuela.

La mayoria de los encuestados se consideró pobre y tenian la percepción de que siempre lo habían sido. Y la noción manifiesta acerca de la pobreza fue la carencia de cosas o dinero para adquirir esas cosas. En El Salvador la edad mínima para trabajar es de 10 años. En el caso de la investigación, existen infantes menores de 10 años que y a se encuentran en el mercado laboral. En lo educativo, la media y la moda era la primaria. Los analfabetos mayores de 10 años representaron cerca del $35 \%$.

Aspectos familiares. Relacionados con la desintegración familiar, las uniones libres, acompañados a temprana edad y el predominio de mujeres como jefas de hogar. En este aspecto, los resultados obtenidos fue población joven e infantil, no existieron porcentajes significativos de población casada y acompañada. En cambio, si se encontró hogares donde las mujeres eran las jefas del hogar.

Aspectos económicos, aquellos aspectos referidos al ingreso, ahorro y empleo. Representados por ingresos bajos, trabajo no especializado, nulo ahorro, uso de ropa y muebles de segunda mano (en algunos casos, carecer de muebles o bienes esenciales), costumbres de empeñar; cuando se riene dinero se gasta bastante sin importar que después no se tenga.

Complementando la información sobre el ingreso y los niveles de pobreza, la mayoría de las personas vivía en lo propio, no obstante, carecian de muchas cosas de la vida cotidiana. Cerca de la mitad no tenía bienes de primera 
necesidad como cocina, utensilios de cocina, muebles de sala y comedor. Aquellos grupos familiares que sí poseian bienes, no los tenían completos.

$Y$, ¿En qué condiciones de vivienda habitaban? En primer lugar, la situación de la vivienda del país cambió sustancialmente después de los terremotos acaecidos en el 2001. Sin cuantificar las perdidas humanas, se destruyeron alrededor de $100 \mathrm{mil}$ viviendas y se dañaron $25 \mathrm{mil}$, incrementando asíel déficit habitacional del país. En segundo lugar, el tipo de vivienda al que rienen acceso las personas carecen de los espacios adecuados, de los servicios básicos completos (agua, energía eléctrica, alcantarilla, otros), y son construcciones de materiales de baja calidad. Esto hace que la crisis habitacional salvadoreña se vuelva mas crítica y estructural, precisamente porque las nuevas construcciones habitacionales populares, además de una habitación y a lo sumo dos, con casi nulo espacio de área de pario, son construidas con un área de 40 y $50 \mathrm{mts}^{2}$ respectivamente.

El resultado a esperar es precisamente que exista un alto porcentaje de hogares que vive en estas condiciones de hacinamiento; y así fue, la mayoría de los hogares vivía en lo propio, pero residfa en una casa de una sola habitación con o sin división, encontrando un mínimo de una persona por hogar a un máximo de 16. En tres comunidades (La Unión, San Vicente y San Martín) tenian los servicios sanitarios, pero el $58 \%$ carecian de alcantarillado y de conductos de aguas negras.

El ambiente, los recursos y la educación, también se relacionan a otro aspecto de la vida del ser humano: su salud, que en muchas mediciones tradicionales de pobreza queda fuera. No obstante, el PNUD lo considera de manera indirecta a través de la esperanza de vida, indicador que se incluye en su Indice de Desarrollo Humano. La edad promedio de los hogares encuestados fue de 27 años, la mínima fise de menos de un año y la máxima de 99 años, exisciendo un predominio de mayor población joven en mujeres que en hombres. Siendo las enfermedades más comunes las respiratorias. En relación a las condiciones físicas y emocionales, la población mostró tener condiciones aceptables, si bien no practicaban acrividades fisicas, no consumian drogas, ni analgésicos, ni alcoholy tabaco.

\section{Conclusiones generales}

Existe un círculo vicioso que profundiza los niveles de pobreza en las personas: bajo nivel educativo y alto nivel de cultura de pobreza que los lleva a trabajos no cualificados, que a su vez, trae como consecuencia baja remuneración y desencadena un bajo poder adquisicivo o el poco acceso a bienes y servicios, tal y como lo manifestaron los entrevistados.

Las características de los pobres en E Salvador, y de acuerdo a los datos obtenidos en la investigación, son los siguientes: desde el punto de vista del método del ingreso, existe un alto porcentaje de pobres en El Salvador. Desde el punto de vista de acceso a bienes, existió un alto porcentaje de la población que relacionó la pobreza con la carencia de bienes, es mas, de acuerdo a los resultados, muy pocas familias tenían propiedades adicionales a la vivienda, un poco menos de la mitad no tenía bienes domésticos y más de la mitad no los tenia completos.

Es innegable la necesidad urgente de políticas socio-económicas que contribuyan a mejorar las condiciones de vida en las diferentes áreas, no sólo por los resultados obrenidos, sino además por lo manifestado por la población. El 25,8\% dijo que necesitaba un mejor trabajo para mejorar su situación y para que éste exista es necesario que se abran fuentes de trabajo que por sí solos no se van a producir.

Existió una diferencia entre la población del municipio de $\mathrm{La}$ Unión y el resto. Las características en cuanto percepción o pensamiento, nivel educativo, empleo, acceso a bienes, niveles de pobreza fueron similares al resto, sin embargo la pobreza se relaciona con el narcotráfico, el ocio y el acomodamiento de las personas a esperar a realizar venta de droga.

Una de las riquezas mayores de los resultados en la investigación fue la accitud de las personas en relación de ellos mismos y en su concepción de pobreza. Fue interesante visualizar las diferentes concepciones de pobreza que van desde los que la conciben como carencia de algo, a los que prefieren verla como herencia, sin menospreciar aquellos que buscan la respuesta de su sicuación con Dios. La mayoría de estas concepciones constituyen formas de justificar su realidad ante la impotencia de no poder cambiarla.

\section{Citas}

PNUD. "Inf orme de Desarzollo Humano. El Salvador 2003. Desafios y Opciones en tiempos de globalizaciơn". Pág. 49

PNUD. "E] Salvador: Estadisticas relacionadas objecivos y metas de desarrollo del milenio". En linea, htip://www.pnud.org-sv/-desarrollohumano/hdr2003/ IMDHES2003.doc 